\title{
BCAR4 induces antioestrogen resistance but sensitises breast cancer to lapatinib
}

\section{MFE Godinho', JD Wulfkuhle ${ }^{2}$, MP Look ${ }^{3,4}$, AM Sieuwerts ${ }^{3,4}$, S Sleijfer ${ }^{3,4}$, JA Foekens ${ }^{3,4}$, EF Petricoin III2, LCJ Dorssers' and T van Agthoven ${ }^{*, 1}$}

'Department of Pathology, Josephine Nefkens Institute, Erasmus MC-University Medical Center Rotterdam, Room Be 432A, PO Box 2040, Rotterdam 3000 CA, The Netherlands; ${ }^{2}$ Center for Applied Proteomics and Molecular Medicine, George Mason University, Manassas, VA 20I I 0, USA; ${ }^{3}$ Department of Medical Oncology, Josephine Nefkens Institute, Erasmus MC-University Medical Center Rotterdam, Rotterdam 3000 CA, The Netherlands; ${ }^{4}$ Cancer Genomics Center, Josephine Nefkens Institute, Erasmus MC-University Medical Center Rotterdam, Rotterdam 3000 CA, The Netherlands

BACKGROUND: High BCAR4 and ERBB2 mRNA levels in primary breast cancer associate with tamoxifen resistance and poor patient outcome. We determined whether BCAR4 expression sensitises breast cancer cells to lapatinib, and identifies a subgroup of patients who possibly may benefit from ERBB2-targeted therapies despite having tumours with low ERBB2 expression.

METHODS: Proliferation assays were applied to determine the effect of BCAR4 expression on lapatinib treatment. Changes in cell signalling were quantified with reverse-phase protein microarrays. Quantitative reverse-transcriptase polymerase chain reaction (RT-PCR) of ERBB2 and BCAR4 was performed in 1418 primary breast cancers. Combined BCAR4 and ERBB2 mRNA levels were evaluated for association with progression-free survival (PFS) in 293 oestrogen receptor- $\alpha$ (ER)-positive patients receiving tamoxifen as first-line monotherapy for recurrent disease.

RESULTS: BCAR4 expression strongly sensitised ZR-75-I and MCF7 breast cancer cells to the combination of lapatinib and antioestrogens. Lapatinib interfered with phosphorylation of ERBB2 and its downstream mediators AKT, FAK, SHC, STAT5, and STAT6. Reverse transcriptase-PCR analysis showed that $27.6 \%$ of the breast cancers were positive for BCAR4 and $22 \%$ expressed also low levels of ERBB2. The clinical significance of combining BCAR4 and ERBB2 mRNA status was underscored by the finding that the group of patients having BCAR4-positive/ERBB2-low-expressing cancers had a shorter PFS on tamoxifen treatment than the BCAR4-negative group.

CONCLUSION: This study shows that BCAR4 expression identifies a subgroup of ER-positive breast cancer patients without overexpression of ERBB2 who have a poor outcome and might benefit from combined ERBB2-targeted and antioestrogen therapy. British Journal of Cancer (2012) I 07, 947-955. doi:I0.1038/bjc.20I2.35 I www.bjcancer.com

Published online 14 August 2012

(c) 2012 Cancer Research UK

Keywords: BCAR4; ERBB2; targeted therapy; breast cancer; tamoxifen resistance

Tamoxifen has an important role in the treatment of patients with oestrogen receptor- $\alpha$ (ER)-positive primary breast cancer, both in the adjuvant and metastatic setting (Davies et al, 2011). Its efficacy is limited by primary (intrinsic) or secondary (acquired) resistance. A better understanding of the mechanisms involved is required to overcome resistance and for developing more effective therapies. Several genes and mechanism causing antioestrogen resistance were identified (Dorssers and Veldscholte, 1997; Van Agthoven et al, 1998; Brinkman et al, 2000; Massarweh and Schiff, 2007; Riggins et al, 2007; Musgrove and Sutherland, 2009; Barone et al, 2010; Van Agthoven et al, 2010), including the novel breast cancer antioestrogen resistance 4 (BCAR4) gene (Meijer et al, 2006). Ectopic expression of BCAR4 causes antioestrogen resistance, anchorage independence, and tumour growth in nude mice (Meijer et al, 2006; Godinho et al, 2011). BCAR4 mRNA is detected in $22-29 \%$ of primary breast cancers. High levels are associated with shorter progression-free survival (PFS) in patients treated with tamoxifen for recurrent disease, and associate with poor

*Correspondence: Dr T van Agthoven; E-mail: a.vanagthoven@erasmusmc.nl Received 17 April 2012; revised 12 July 2012; accepted 13 July 2012; published online 14 August 2012 metastasis-free survival (MFS) and overall survival (OS), reflecting tumour aggressiveness (Godinho et al, 2010).

BCAR4 has been found in several mammalian species, being well conserved in higher primates (Meijer et al, 2006; Godinho et al, 2011). In the functional screening for genes causing tamoxifen resistance, it was isolated from a human placenta cDNA library only (Meijer et al, 2006; Godinho et al, 2011). Searches in public expression databases and in the literature showed that high BCAR4 expression is only found in placenta and the oocyte (Meijer et al, 2006; Godinho et al, 2011). In other normal adult tissues, expression of BCAR4 was not found. The species and tissuespecific expression strongly indicates a role for BCAR4 in mammalian early development and pregnancy. Surprisingly, the $B C A R 4$ gene is absent in the mouse and rat (Godinho et al, 2011). Important differences exist between human and mouse placental development and function. In the mouse, in contrast to the human situation, trophoblast implantation is superficial, the transformation of the uterine arteries depends on maternal factors, and mouse placenta produces fewer placental hormones (Malassine et al, 2003; Carter, 2007). At this point it is only possible to speculate on the function of this gene, but it is likely that differences in placental development and function could explain the 
absence in these organisms. BCAR4 may have a function in placenta and early development, therefore it cannot be excluded that in mouse and rat its function has been taken over by other genes.

BCAR4-induced tamoxifen resistance depends on the presence of ERBB2 (HER2) and ERBB3 receptors (Godinho et al, 2010). We hypothesised that BCAR4 expression may sensitise breast cancer cells to the small-molecule tyrosine kinase activity inhibitor of EGFR and ERBB2. In this study, BCAR4-expressing cells were assessed for their sensitivity to lapatinib, given alone and in combination with antioestrogens. In addition, the effects of treatment on ERBB2 and ERBB3 downstream signalling were measured. As increased ERBB2 activity has been associated with resistance to cytotoxic agents in breast cancer, the impact of $B C A R 4$ expression on sensitivity to several cytotoxic drugs was assessed. The results of our cell line studies showed that ectopic expression of BCAR4 results in activation of the ERBB2 signalling pathway without overexpression of ERBB2. Therefore, we determined the incidence of breast cancers expressing BCAR4 and low $E R B B 2$ levels, and how this group of patients fares when treated with tamoxifen for advanced disease.

\section{MATERIALS AND METHODS}

\section{Cell lines and culture conditions}

ZR-75-1 and MCF7 cell lines were kind gifts of RJB King (ICRF, London) and RB Dickson (NCI, Bethesda), respectively. Cell lines were initially authenticated by karyotyping, and in November 2011 using the AmpFlSTR Identifiler Direct PCR Amplification Kit (Applied Biosystems International, Nieuwerkerk a/d Ijssel, The Netherlands). Cell lines derived from the breast cancer cell line ZR-75-1 containing empty vector, or expression constructs with BCAR4 (Meijer et al, 2006), BCAR1 (Brinkman et al, 2000), BCAR3 (Van Agthoven et al, 1998), or EGFR (Van Agthoven et al, 1992), and MCF7 breast cancer cells with a construct containing BCAR4 were cultured as previously described (Van Agthoven et al, 1998).

\section{Drug sensitivity assays}

Cells were seeded in 96-well plates at a density of 5000 cells per well in $100 \mu \mathrm{l}$ RPMI 1640 medium (Invitrogen, Breda, The Netherlands). After $24 \mathrm{~h}$, serial dilutions of lapatinib (GlaxoSmithKline, Stevenage, UK), doxorubicin (Pharmachemie B.V., Haarlem, The Netherlands), 5-fluorouracil (EBEWE Pharma, Unterach, Austria), methotrexate (Emthexate PF, Pharmachemie B.V.), ifosfamide (Holoxan, Baxter B.V., Utrecht, The Netherlands), or paclitaxel (Paclitaxel, EBEWE Pharma) were added. All drugs were tested in combination with $17 \beta$-oestradiol or 4-hydroxytamoxifen (SigmaAldrich Chemie, Zwijndrecht, the Netherlands) or ICI182,780 (Zeneca Pharmaceuticals, Macclesfield, UK). To assay the effects of oestrogen, cells were seeded at a density of 5000 cells per well in $100 \mu$ l RPMI 1640 without phenol red, supplemented with $6 \%$ heatinactivated bovine calf serum (Hyclone, Logan, UT, USA). Twentyfour hours after seeding, $100 \mu \mathrm{l}$ of medium containing $0.01,0.1$, or $1 \mu \mathrm{m}$ lapatinib and increasing concentrations of oestradiol were added. WST-1 proliferation assays (Roche Diagnostics, Almere, The Netherlands) were performed on ZR-75-1- or MCF7-derived cell lines after 5 or 6 days, respectively. $\mathrm{IC}_{50}$ values were estimated by sigmoid inhibitory effect models 107 and 108 as implemented in the software programme Phoenix WinNonLin 6.1 (Pharsight, Mountain View, CA, USA).

\section{Inhibition of gene expression by small interfering (si)RNAs}

Transfections with HiPerfect (Qiagen, Venlo, The Netherlands) were performed according to the manufacturer's instructions. Small interfering RNAs were On TARGETplus-SMARTpools, each consisting of three different oligonucleotides: EGFR (L-003114-000005), ERBB2 (L-003126-00-005), ERBB3 (L-003127-00-0005), and ERBB4 (L003128-00-0005; Dharmacon, Perbio-Science, Etten Leur, The Netherlands). Final concentration of siRNA was $5 \mathrm{~nm}$. WST-1 assays were performed after 6 days.

\section{Reverse-phase protein microarrays}

Cells cultured in oestradiol- or 4-hydroxytamoxifen-containing medium were treated without or with 0.01 or $0.1 \mu \mathrm{m}$ lapatinib for $17 \mathrm{~h}$. Cells were rinsed with ice-cold DPBS (Gibco, Invitrogen), and lysed with pre-heated $\left(75^{\circ} \mathrm{C}\right)$ extraction buffer consisting of equal parts of T-PER (Pierce, Thermo Scientific, Etten-Leur, The Netherlands) and Tris-Glycine-SDS Sample Buffer (Invitrogen) containing PhosSTOP Phosphatase Inhibitors, Complete Mini Protease Inhibitors (Roche Diagnostics), and $4 \%$ of $\beta$-mercaptoethanol (Merck, Schiphol-Rijk, The Netherlands). Lysates were boiled for $8 \mathrm{~min}$ and stored at $-80^{\circ} \mathrm{C}$. Reverse-phase protein microarray analysis was performed as described (Van Agthoven et al, 2012). A list of antibodies used is presented in Supplementary Table 1.

\section{Patient samples}

ERBB2 and BCAR4 mRNA levels were measured in 1418 ER-positive and negative primary breast cancers as described in Van Agthoven et al (2009) and Godinho et al (2010). Here we assessed the prognostic and predictive values of a combined BCAR4 and ERBB2 status. BCAR4 and ERBB2 were determined according to the definitions/cut points in the aforementioned studies. To determine the association of the combination of BCAR4 and ERBB2 mRNA levels and PFS, 293 samples from patients with ER-positive cancers who received tamoxifen treatment as first-line therapy for metastatic disease were analysed. The associations of the combined BCAR4 and ERBB2 levels with tumour aggressiveness in terms of MFS and OS were determined on 497 ER-positive cancers from patients with lymph node-negative disease. None received systemic adjuvant therapy. Statistical analyses were performed as previously detailed (Godinho et al, 2010).

\section{Quantification of gene expression}

RNA isolation of cell lines, complementary DNA synthesis, normalisation to reference genes and quantification were performed as described (Sieuwerts et al, 2005; Van Agthoven et al, 2009; Godinho et al, 2010). TaqMan gene expression assays for EGFR-Hs01076091_m1, ERBB2-Hs00170433_m1, ERBB3Hs00176538_m1, ERBB4-Hs00171783_m1, and BCAR4Hs00415922_m1 were used according the recommendations of the supplier Applied Biosystems International.

\section{RESULTS}

Inhibition of ERBB2/3 expression abrogates BCAR4induced antioestrogen-resistant proliferation

Previously we have shown that BCAR4-induced tamoxifenresistant proliferation of ZR-75-1 cells depends on the presence of ERBB2 and ERBB3 (Godinho et al, 2010), while ERBB2 is not overexpressed or amplified in this cell line (Hollestelle et al, 2010). In MCF7 cells, we investigated whether BCAR4 expression also induces ERBB2/3-mediated proliferation. The expression of the four ERBB receptors were inhibited with siRNAs, in the absence or presence of the pure antioestrogen ICI182,780. In contrast to 4-hydroxytamoxifen, this antioestrogen fully inhibits growth of wild-type MCF7 cells. Inhibition of mRNA transcripts was verified by quantitative real-time reverse transcriptase polymerase chain 

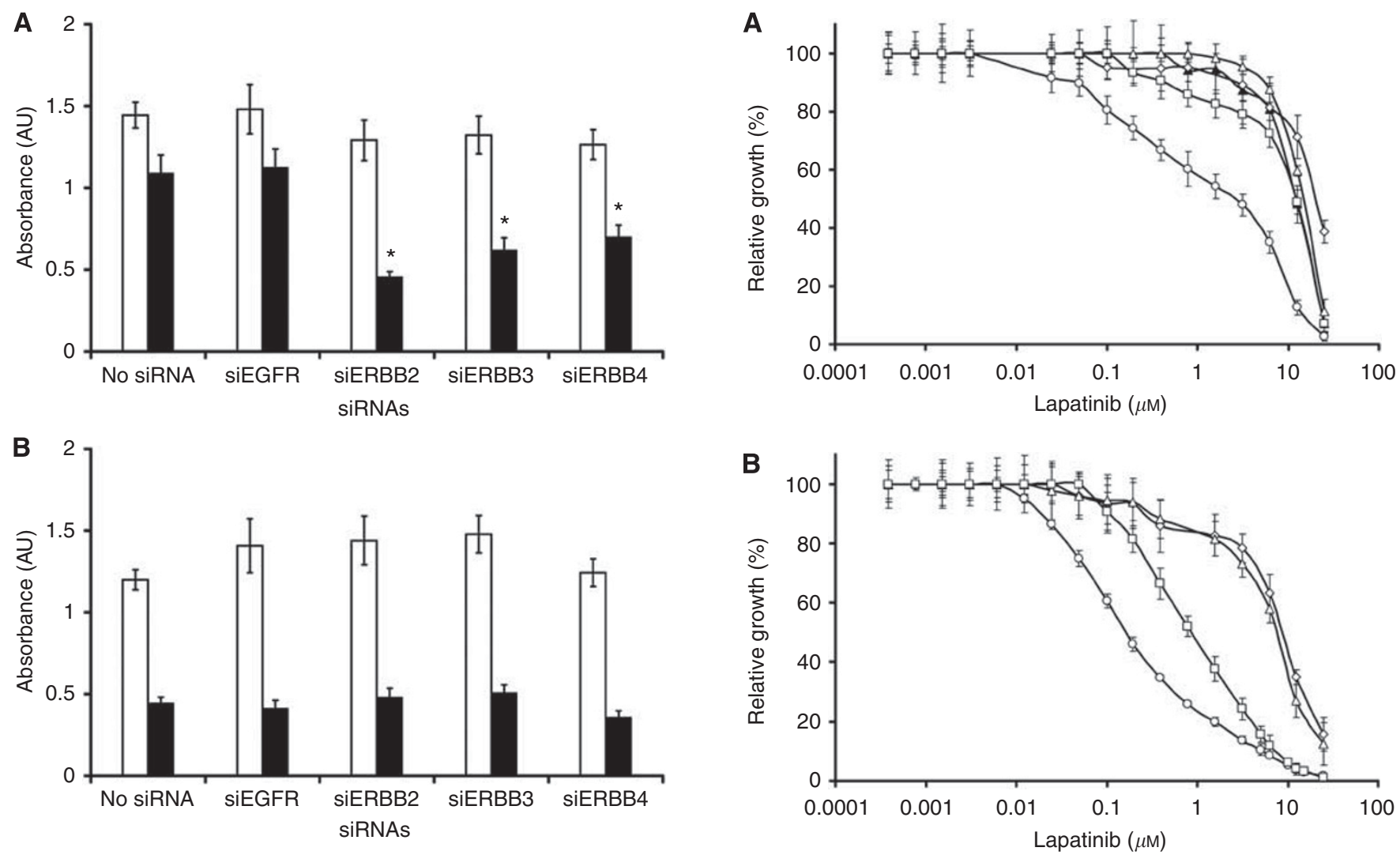

Figure I Knockdown of ERBB receptors reduces proliferation of antioestrogen-resistant MCF7/BCAR4 cells. MCF7/BCAR4 (A) and MCF7/vector (B) cells were cultured in the absence (open bars) or presence (closed bars) of the antioestrogen ICII82,780. The inhibition of ERBB receptors by specific siRNAs was measured with a proliferation assay. Average of five replicates and SDs are shown. Significance was determined by the Mann-Whitney $U$-test. $* P<0.05$, compared with cells cultured without siRNAs. Abbreviation: $A U=$ arbitrary units.

reaction (RT-PCR, and was more than $70 \%$ for $E G F R, 88 \%$ for $E R B B 2,66 \%$ for $E R B B 3$, and $75 \%$ for $E R B B 4$.

In foetal bovine serum-containing medium, the proliferation capacity of MCF7 cells expressing BCAR4 (MCF7/BCAR4) and MCF7 vector-containing cells was not affected by the inhibition of the expression of the $E R B B$ receptors (Figure 1A and B). Similarly to BCAR4 expression in ZR-75-1 cells, MCF7/BCAR4 cells were antioestrogen resistant and able to grow in the presence of ICI182,780 (Figure 1A). Under this culture condition, the inhibition of $E R B B 2, E R B B 3$, and $E R B B 4$ expression resulted in decreased cell proliferation, indicating that also in MCF7/BCAR4 cells, ERBB signalling is involved in antioestrogen resistance. Growth of MCF7/vector cells was fully inhibited by ICI182,780, and inhibition of the ERBB receptor expression had no further effect (Figure 1B).

\section{BCAR4 expression increases the sensitivity of cells to lapatinib}

We speculated that BCAR4 expression may increase the sensitivity to the EGFR/ERBB2 tyrosine kinase inhibitor lapatinib. Sensitivity to lapatinib was determined in ZR-75-1 cells containing empty expression vector (ZR/vector) or BCAR4 (ZR/BCAR4). Cells expressing BCAR1 (ZR/BCAR1), BCAR3 (ZR/BCAR3), or EGFR (ZR/EGFR) were used for comparison. These latter genes were shown to induce tamoxifen resistance by mechanisms independent of ERBB2 and ERBB3 (Van Agthoven et al, 1992, 1998; Brinkman

Figure 2 BCAR4 sensitises ZR-75-I cells to lapatinib. ZR/vector control $(\boldsymbol{\Delta})$, ZR/BCAR4 $(\bigcirc)$, ZR/BCARI $(\diamond)$, ZR/BCAR3 $(\boldsymbol{\Delta})$ or ZR/EGFR cells $(\square)$ were plated in oestradiol-containing medium $(\mathbf{A})$, or 4-hydroxytamoxifen-containing medium (B) with increasing doses of lapatinib as indicated. Concentrations of lapatinib ( $X$ axis) are presented on a logarithmic scale. Results are expressed as a percentage of maximal growth as measured with a WST-I proliferation assay. Average of five replicates and SDs are presented.

et al, 2000; Meijer et al, 2006). Titration experiments showed that cells expressing BCAR4 were the most sensitive to lapatinib in the presence of oestradiol (Figure $2 \mathrm{~A}$ ). The $\mathrm{IC}_{50}$ values for ZR/BCAR4 cells were 10 - to 20 -times lower than the $\mathrm{IC}_{50}$ determined for the other cell lines. In the presence of oestradiol and lapatinib expression of BCAR1, BCAR3, or EGFR had no impact on proliferation, which was similar to the empty vector-containing cells.

Lapatinib sensitivity was also determined in MCF7 cells. In the presence of fetal bovine serum alone, the determined $\mathrm{IC}_{50}$ values for MCF7/BCAR4 cells were similar to the $\mathrm{IC}_{50}$ values determined for MCF/vector cells (7-9 $\mu \mathrm{M}$ and 8-12 $\mu \mathrm{M}$, respectively). This is in agreement with the inhibition of the ERBB receptors having no effect on proliferation of MCF7/BCAR4 under this culture condition (Figure 1). Under these culture conditions, the cells apparently depend on the ER pathway for proliferation.

\section{Antioestrogens enhance the sensitivity of BCAR4- expressing cells to lapatinib}

We tested whether antioestrogens could enhance the sensitivity to lapatinib. Proliferation of wild-type ZR-75-1 cells is fully inhibited by $1 \mu \mathrm{M} 4$-hydroxytamoxifen in the culture medium. ZR/BCAR4 was also the most sensitive cell line to the combination of lapatinib and 4-hydroxytamoxifen compared with ZR/BCAR1, ZR/BCAR3, or ZR/EGFR cells (Figure 2B). Tamoxifen further increased the sensitivity of ZR/BCAR4 cells to lapatinib by approximately 
three-fold. A very similar lapatinib dose-response curve was obtained with the presence of ICI182,780 (data not shown).

ZR-75-1 cells with forced expression of the EGFR are tamoxifen resistant and oestrogen-independent in the presence of $10 \mathrm{ng} / \mathrm{ml}$ of EGF (Van Agthoven et al, 1992). Compared with oestradiolstimulated cultures, ZR/EGFR cells were six-fold more sensitive than controls to the combination of lapatinib, 4-hydroxytamoxifen, and EGF. ZR/BCAR1 and ZR/BCAR3 cells showed similar lapatinib dose-response curves in oestradiol and 4-hydroxytamoxifen-containing medium, approximately 35 -fold less sensitive than ZR/BCAR4 cells. Growth of ZR/vector cells was fully inhibited by 4-hydroxytamoxifen, therefore the sensitivity to lapatinib under this culture condition is not informative (data not shown). ICI182,780 increased the sensitivity of MCF7/BCAR4 cells to lapatinib by approximately 10 -fold.

\section{Lapatinib inhibits ERBB2 signalling in BCAR4-expressing cells}

Reverse-phase protein microarray analysis was used to determine the effects of lapatinib treatment on the levels of 68 total or phosphorylated proteins having a role in survival, motility, death, growth, metabolism, and inflammation (Supplementary Table 2). To circumvent the problem that changes in phosphorylation were solely due to toxicity, cells were cultured in medium without lapatinib or with low doses of 0.01 or $0.1 \mu \mathrm{M}$ lapatinib for $17 \mathrm{~h}$. These concentrations resulted in limited growth inhibition after 5 days in culture (Figure $2 \mathrm{~A}$ and B). Lapatinib treatment had no prominent effects on protein phosphorylation in ZR/vector, ZR/ BCAR1, ZR/BCAR3, or ZR/EGFR cells, while clear changes were observed for ZR/BCAR4 cells (Figure 3A). We quantified the effects on the phosphorylation of its target, the ERBB2 receptor, the ERBB3 receptor, and several downstream mediators. ZR/BCAR4 cells do not express EGFR (Van Agthoven et al, 2012), therefore changes caused by the addition of lapatinib cannot be attributed to this pathway.

As observed before (Van Agthoven et al, 2012) in oestradiolcontaining medium, phosphorylation of ERBB2 (Tyr1248) was 12 -fold higher in ZR/BCAR4 cells compared with the phosphorylation levels in control cells (Figure 3B). Under this culture condition, $0.01 \mu \mathrm{M}$ lapatinib completely inhibited ERBB2 phosphorylation. The combination of 4-hydroxytamoxifen and $0.1 \mu \mathrm{m}$ lapatinib resulted in a two-fold decrease in ERBB2 phosphorylation (Figure 3B). Lapatinib exerted no effect on ERBB2 phosphorylation in the other cell lines.

ZR/BCAR4 cells showed the highest levels of phosphorylated ERBB3 (Tyr1289; Figure 3C; Van Agthoven et al, 2012). Lapatinib treatment in oestradiol-containing cultures resulted in moderately decreased ERBB3 phosphorylation, but had no effect in the presence of 4-hydroxytamoxifen. In the other cell lines, lapatinib did not modulate ERBB3 phosphorylation (Figure 3C). Phosphorylation of several downstream mediators, such as AKT (Ser473), FAK (Tyr576-577), SHC (Tyr317), STAT5 (Tyr694), and STAT6 (Tyr349), was higher in ZR/BCAR4 cells compared with control cell lines (Figure 3D-H). Similar to the effect on ERBB2 phosphorylation, in oestradiol-containing medium, $0.01 \mu \mathrm{m}$ lapatinib inhibited phosphorylation of these downstream mediators. In medium containing 4-hydroxytamoxifen, a higher dose of lapatinib was needed to reduce phosphorylation levels. In the remaining cell lines, lapatinib treatment had little or no effect on the phosphorylation of these signalling molecules.

\section{BCAR4-expressing cells alternate between signalling pathways to survive}

Proliferation of ZR/BCAR4 cells was more sensitive to the combination of lapatinib and 4-hydroxytamoxifen than to the combination of lapatinib and oestradiol (Figure 2). Moreover, phosphorylation of ERBB2 and downstream targets is inhibited in the presence of oestradiol and lapatinib. The addition of 4-hydroxytamoxifen increased ERBB2 levels and downstream signalling. Therefore, we hypothesised that if the ERBB2 signalling pathway is inhibited by lapatinib, BCAR4-expressing cells may switch to the ER pathway to sustain survival and proliferation. To test this, we analysed the effects of lapatinib treatment on oestradiol dependence in short-term cultures. While ZR/BCAR4 cells showed maximal proliferation capacity in the absence of oestradiol (Figure 4A), ZR/vector cells required supplementation of $10-100 \mathrm{pm}$ of oestradiol. Figure $4 \mathrm{~B}$ shows that in the presence of 0.01 or $0.1 \mu \mathrm{m}$ lapatinib, oestrogen dependence of $\mathrm{ZR} / \mathrm{vector}$ cells remained unchanged. In contrast, proliferation of ZR/BCAR4 cells was less inhibited by lapatinib in the presence of more than $10 \mathrm{pM}$ of oestradiol (Figure 4A). These results indicate that ZR/BCAR4 cells can evade the growth inhibitory effects of lapatinib in part through ER signalling.

\section{BCAR4 and chemotherapy}

As several studies indicate an association between ERBB2 overexpression and resistance to chemotherapy (reviewed in Tan and $\mathrm{Yu}, 2007)$, and BCAR4 expression enhances ERBB2 signalling, we determined the sensitivity of BCAR4-expressing cells to drugs currently included in common breast cancer treatment regimens. To investigate alterations in drug sensitivity, cells were cultured in oestradiol- or 4-hydroxytamoxifen-containing medium and increasing concentrations of the different chemotherapeutics.

As a typical example, a dose-response curve of $\mathrm{ZR} /$ vector, ZR/BCAR4, ZR/BCAR1, ZR/BCAR3, and ZR/EGFR cells to methotrexate is shown in Supplementary Figure S1. No major differences in sensitivity to the drug between the different cell lines, either in the presence of oestradiol (Supplementary Figure S2A) or 4-hydroxytamoxifen (Supplementary Figure S2B), were observed. Similar results were obtained for ifosfamide, 5-fluorouracil, doxorubicin, and paclitaxel, indicating no changes in sensitivity of conventional drugs due to the expression of BCAR4. Moreover, no major differences were found between the $\mathrm{IC}_{50}$ values determined for all the BCAR cell lines and for the control cells (Supplementary Table 3), with exception of ZR/ BCAR1 cells being less sensitive to doxorubicin, and ZR/EGFR cells being less sensitive to doxorubicin and 5-fluorouracil.

\section{BCAR4 mRNA levels may define a subgroup of patients who are eligible for treatment with established ERBB2 inhibitors}

At present, only patients with breast cancers overexpressing ERBB2 or with gene amplification are eligible for ERBB2-targeted therapies. Our functional in vitro studies show that BCAR4 activates the ERBB2 pathway yielding resistance against antioestrogens in cell lines not overexpressing ERBB2. This could imply that BCAR4 expression identifies an additional subgroup of patients with activated ERBB2, but lacking ERBB2 overexpression. To investigate the prevalence of this group, both BCAR4 and $E R B B 2$ mRNA status were determined in a large cohort of primary breast cancers previously measured by RT-PCR (Van Agthoven et al, 2009; Godinho et al, 2010). BCAR4 was detected in 392 out of $1418(27.6 \%)$ samples (Figure 5A). High expression of ERBB2 was detected in 233 specimens (16.4\%). Among the BCAR4-positive samples, 80 had high and 312 had low expression of ERBB2, indicating the existence of a group of patients (22\%) with BCAR4positive cancers and low expression of ERBB2.

\section{Clinical relevance of combined BCAR4 and ERBB2 status}

$B C A R 4$ and $E R B B 2$ have been found to be independently predictive for tamoxifen resistance in recurrent breast cancer. While ERBB2 
A
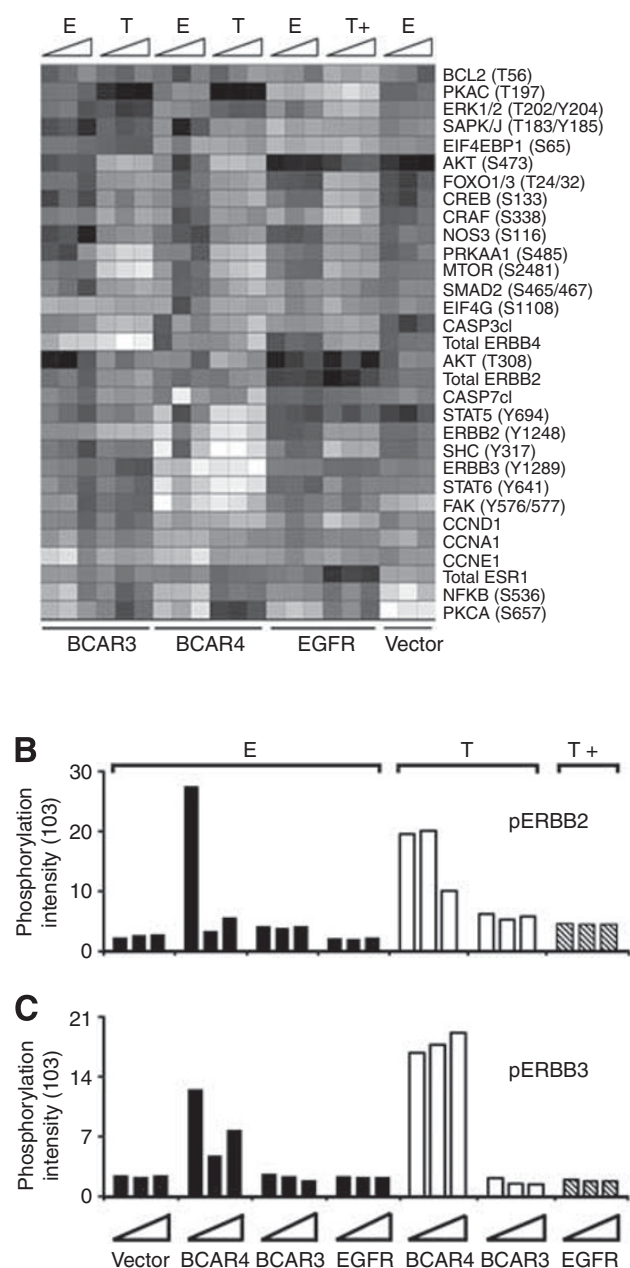
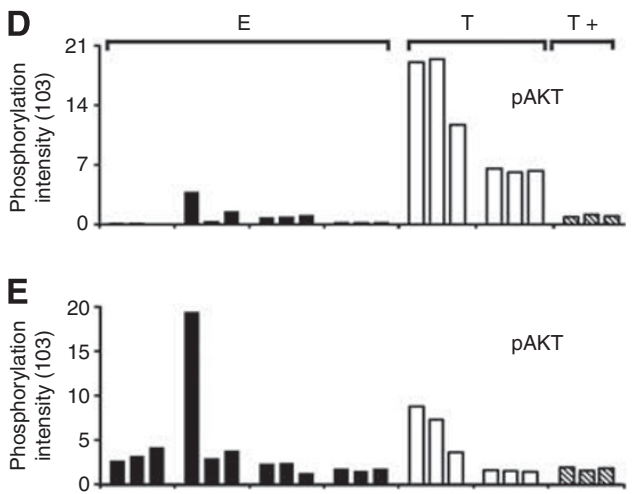

$\mathbf{F}$

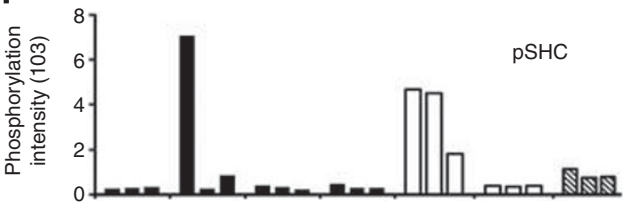

G

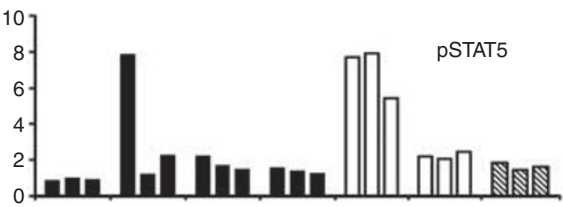

H

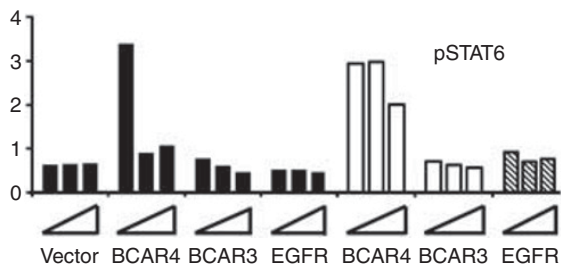

Figure 3 Lapatinib treatment inhibits ERBB2 and ERBB3 signalling in ZR/BCAR4 cells. (A) Molecular network analysis of ZR-75-I-derived antioestrogenresistant cell lines (horizontal axis) treated with lapatinib. Lysates were analysed with reverse-phase protein microarrays. The heatmap presents the different total and phosphorylated proteins $(n=31$; vertical axis) that showed at least two-fold difference with the vector control cultured in the presence of oestradiol (colour version in Supplementary Figure I and protein data in Supplementary Table I). Higher relative levels are represented in white; lower levels in black. Cells were cultured with oestradiol $(\mathbf{E})$ or 4-hydroxytamoxifen $(T)$, or $T$ and EGF $(T+)$ and treated for $17 \mathrm{~h}$, without, or with 0.01 or $0.1 \mu \mathrm{M}$ lapatinib (triangles represent increasing lapatinib concentrations, from left to right). (B-H) Effects of lapatinib treatment on ERBB2 and ERBB3 signalling. Phosphorylation intensity (Y axis) of ERBB2 (B), ERBB3 $(\mathbf{C})$, AKT (D), FAK (E), SHC (F), STAT5 $(\mathbf{G})$, and STAT6 (H) in the different cell lines (horizontal axis) is presented. Results of two independently derived pools of transduced cells were averaged. Phosphorylation intensity values for antibody staining were negative control subtracted and normalised for total protein concentration. An average of three measurements is presented. SD values $<2 \%$ across the replicates, the three bars for each cell line represent the three different conditions (no or 0.01 or $0.1 \mu \mathrm{m}$ lapatinib).

was not associated with the natural course of the disease in untreated lymph node-negative ER-positive patients with primary breast cancer, patients with BCAR4-positive tumours had a shorter MFS and OS compared with BCAR4-negative tumours (Van Agthoven et al, 2009; Godinho et al, 2010).

Here we assessed the associations of combined BCAR4 and ERBB2 status and clinical tamoxifen resistance in recurrent breast cancer. mRNA levels of 293 ER-positive primary cancers of patients treated with tamoxifen as first-line therapy for metastatic disease were analysed for association with the length of PFS. The individual clinical associations of BCAR4 and ERBB2 mRNA levels for PFS (Table 1) were in agreement with our previous data (Van Agthoven et al, 2009; Godinho et al, 2010). Univariate Cox regression analysis of the combined mRNA status showed that patients with BCAR4-positive tumours with low levels of ERBB2 had a shorter PFS than patients with BCAR4-negative tumours with low $E R B B 2$ levels $(\mathrm{HR}=1.64, P=0.001$; Table 1). Patients with high ERBB2 levels had the shortest PFS, regardless of BCAR4 status (Table 1). The Kaplan-Meier analysis visualises the different outcomes of the patients stratified according to the combined $B C A R 4$ and $E R B B 2$ status (Figure $5 \mathrm{~B}$ ). In the multivariate analysis, the power of the combination of BCAR4 expression and low levels of $E R B B 2$ was independent of the traditional predictive factors for PFS (BCAR4-negative/ERBB2-low vs BCAR4-positive/ERBB2-low, $\mathrm{HR}=1.50, P=0.011$; Table 1$)$.

To assess the associations of combined BCAR4 and ERBB2 levels and tumour aggressiveness, we analysed mRNA status in 497 primary breast cancers. All patients had ER-positive, lymph nodenegative cancer and did not receive adjuvant systemic therapy, allowing the analysis of the natural course of the disease. The mRNA levels were analysed for association with the end points MFS and OS. Metastasis-free survival in patients with BCAR4positive/ERBB2-low tumours was not significantly different from patients with $B C A R 4$-negative/ERBB2-low tumours. Patients with 
A
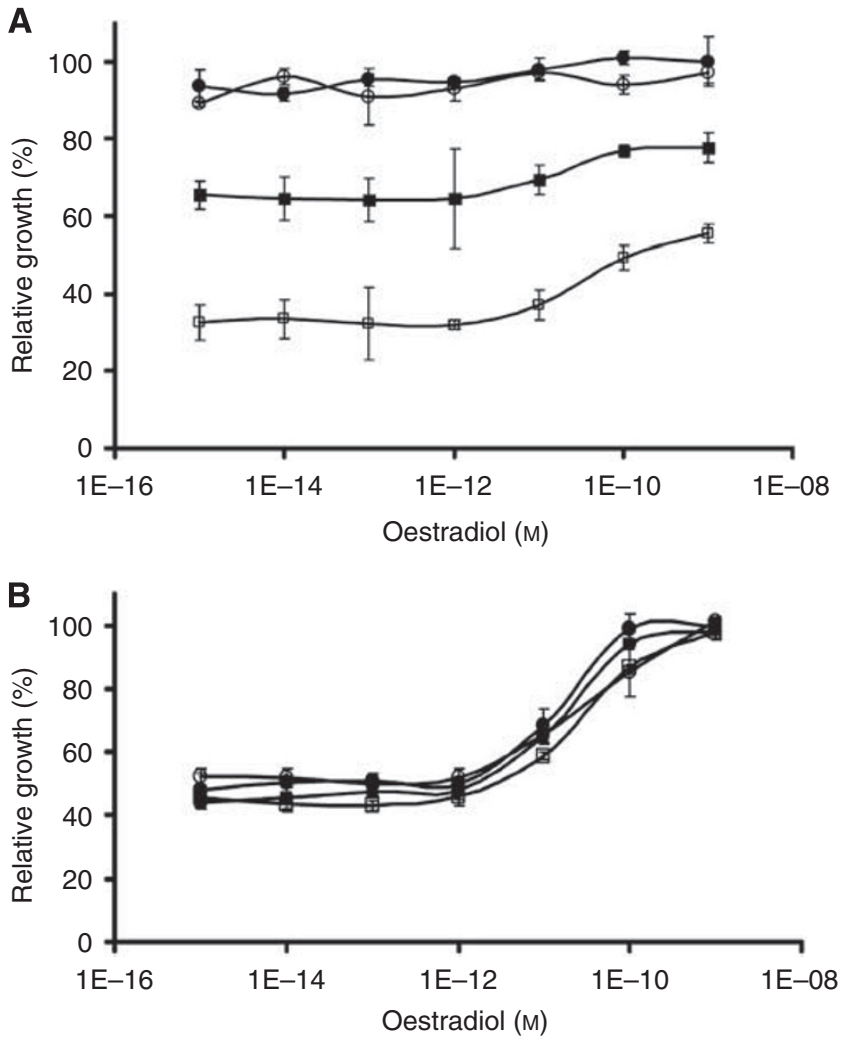

Figure 4 Lapatinib-treated cells utilise ER signalling for survival. ZR/ BCAR4 $(\mathbf{A})$ and ZR/vector $(\mathbf{B})$ cells were plated in the absence $(\mathbf{O})$, $0.01 \mu \mathrm{M}$ lapatinib $(\mathrm{O}), 0 . \mathrm{I}(\mathbf{\square})$, or I $\mu \mathrm{M}(\square)$ of lapatinib and different concentrations of oestradiol, as indicated. Results are expressed as a percentage of maximal growth (cultures with I nM oestradiol but without lapatinib), as measured with a WST-I proliferation assay. Average of three replicates and SD values are presented.

BCAR4-positive/ERBB2-high tumours had the shortest MFS (multivariate $\mathrm{HR}=1.95, P=0.026$; Supplementary Table 4). Analysis for OS indicated that patients with BCAR4-positive/ERBB2low tumours had a significantly shorter OS than patients with $B C A R 4$-negative/ERBB2-low tumours. This difference was independent of the traditional prognostic factors (multivariate $\mathrm{HR}=1.54, P=0.021)$. Patients with BCAR4-positive/ERBB2-high tumours had the shortest OS from all groups (multivariate $\mathrm{HR}=2.25, P=0.004$; Supplementary Table 4 ).

\section{DISCUSSION}

In this study we show that BCAR4 expression sensitises two breast cancer models to lapatinib. As BCAR4 expression in cell lines did not change the sensitivity to different chemotherapeutic agents, the increased sensitivity to lapatinib is not due to a general mechanism of drug resistance. The combination of lapatinib and tamoxifen treatment is more effective at inhibiting breast cancer cell growth than lapatinib alone (Chu et al, 2005; Leary et al, 2010). Also in our BCAR4-expressing cell models, the combination of lapatinib and antioestrogens was more potent in inhibiting cell growth than lapatinib alone; indicating that blocking the ERBB2 pathway with lapatinib re-sensitises BCAR4-expressing cells to antioestrogens.

Breast tumours have been shown to alternate between ER and ERBB2 signalling, and inhibition of one of the pathways reactivated the other (Gutierrez et al, 2005; Lipton et al, 2005; Massarweh and Schiff, 2006; Munzone et al, 2006; Creighton et al,
A
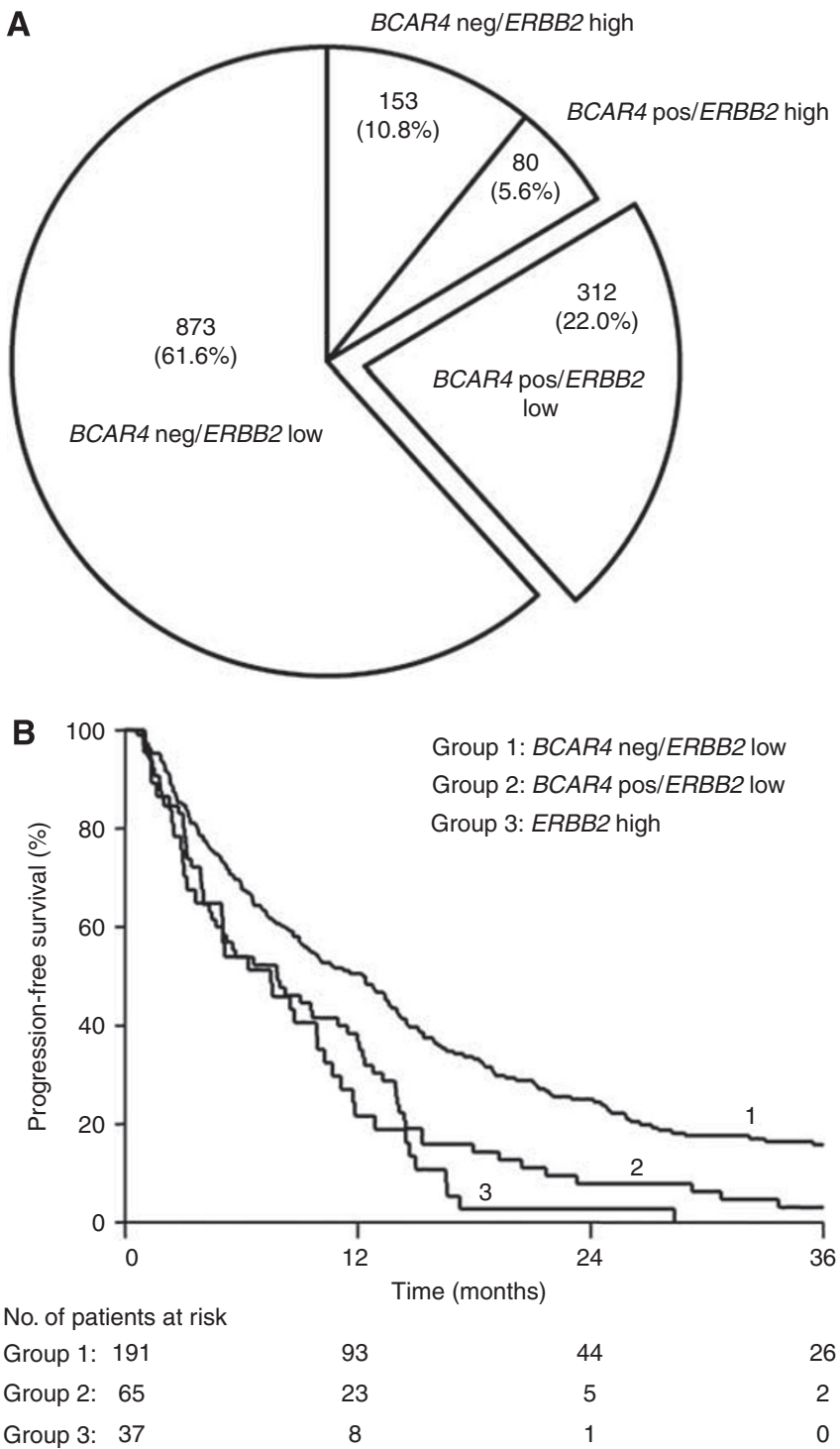

Figure 5 Clinical relevance of combined BCAR4 and ERBB2 status. Expression of BCAR4 and ERBB2 mRNA levels was measured in a cohort of primary breast carcinomas. Patients were stratified according to the combined BCAR4 and ERBB2 status as indicated. BCAR4 was divided in negative (no call), and positive low and high by the mean. There is no relation observed between BCAR4 and ERBB2 expression. (A) Prevalence of breast tumours expressing BCAR4 and low levels of ERBB2. Number of tumours and percentages are shown for each group. (B) Progression-free survival of patients with ER-positive breast cancer who received first-line tamoxifen monotherapy for recurrent breast cancer. Patients with ERBB2high tumours were grouped irrespective of their BCAR4 status. The patients at-risk at 12-month intervals are indicated. Abbreviations: neg $=$ negative, pos $=$ positive.

2008). It has also has been reported that increased ER signalling occurs in lapatinib-treated breast cancer cell lines (Xia et al, 2006; Leary et al, 2010). In the presence of lapatinib and increasing levels of oestradiol, ZR/BCAR4 cells exhibited comparable cell growth kinetics as oestrogen-dependent parental cells (Figure 5A). In culture medium containing lapatinib without oestradiol growth of ZR/BCAR4 cells was strongly inhibited. This suggests that BCAR4 cells use the ER signalling pathway to survive in the presence of low concentrations of lapatinib. Likewise, MCF7/BCAR4 cells cultured in medium without antioestrogens use the ER pathway to 
BCAR4 sensitises breast cancer cells to lapatinib

Table I Associations of combined mRNA levels of BCAR4 and ERBB2 in primary breast tumours with progression-free survival

\begin{tabular}{|c|c|c|c|c|c|c|c|}
\hline \multirow[b]{2}{*}{ PFS } & \multicolumn{4}{|c|}{ Univariate analysis } & \multicolumn{3}{|c|}{ Multivariate analysis } \\
\hline & No. & HR & $95 \% \mathrm{Cl}$ & $\boldsymbol{P}$ & HR & $95 \% \mathrm{Cl}$ & $\boldsymbol{P}$ \\
\hline High vs low & $37 / 256$ & 1.86 & $1.3 \mid-2.65$ & 0.001 & 1.90 & $1.28-2.80$ & 0.001 \\
\hline \multicolumn{8}{|l|}{ BCAR4 } \\
\hline Pos vs neg & $78 / 215$ & 1.57 & $1.20-2.05$ & 0.001 & 1.46 & $1.10-1.93$ & 0.009 \\
\hline \multicolumn{8}{|l|}{ Combined addition } \\
\hline BCAR4 neg/ERBB2 low & 191 & 1 & & & 1 & & \\
\hline BCAR4 pos/ERBB2 low & 65 & 1.64 & $1.23-2.20$ & 0.001 & 1.50 & $1.10-2.04$ & 0.011 \\
\hline BCAR4 neg/ERBB2 high & 24 & 2.17 & $1.40-3.36$ & 0.001 & 2.09 & $1.32-3.29$ & 0.002 \\
\hline BCAR4 pos/ERBB2 high & 13 & 2.03 & $1.15-3.58$ & 0.014 & 2.02 & $1.09-3.73$ & 0.026 \\
\hline
\end{tabular}

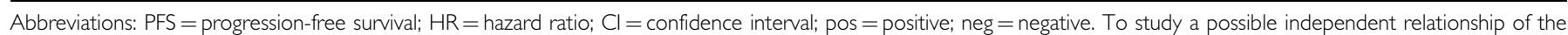

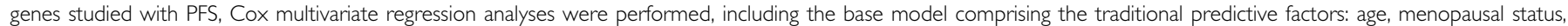

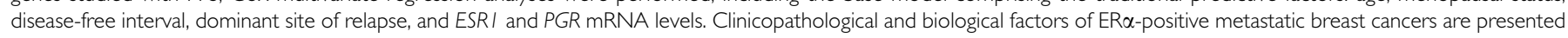
in Supplementary Table 5.

proliferate. These cells are antioestrogen-resistant, but similar to ZR/BCAR4 cells, proliferation is reduced when ERBB2 or ERBB3 are knocked-down. This indicates that MCF7/BCAR4 cells are also dependent on the ERBB2 and ERBB3 pathway to overcome the inhibitory effects of antioestrogens. Apparently, BCAR4 expression enables cells to alternate between signalling pathways to escape the inhibition of one of them.

The mechanism by which BCAR4 activates the ERBB2 and ERBB3 receptors is still unknown, but several hypotheses can be considered. BCAR4 may encode a very small protein, and its predicted anchor signal and two transmembrane domains suggest that the protein is located at cell membranes. Because of its possible location and interaction with ERBB receptors, there is a possibility that BCAR4 may be a target for ADAM proteins, which cleave ERBB ligands (Mochizuki, 2007). This way, cleaved BCAR4 would be free to bind ERBB3, activating ERBB2 and ERBB3 signalling. BCAR4 may interact with ERBB2 through the cell membrane, similar to MUC4 (Carraway et al, 2001). A mechanism similar to nucleolin, which interacts intracellular and activates the ERBB receptors (Di Segni et al, 2008). The hypothesis that BCAR4 may be a secreted protein can also not be excluded. Another possibility is that the BCAR4 protein interacts with and stabilises the ERBB2/ERBB3 dimer, or interferes with the internalisation and/or intracellular transport of the receptors. It has been shown that mucins can influence receptor trafficking and localisation, and because of that, can modulate receptor tyrosine kinase signalling (Funes et al, 2006).

Lapatinib treatment has been shown to prevent ubiquitination and degradation of ERBB2, resulting in the accumulation of inactive receptors at the plasma membrane (Scaltriti et al, 2009). Exposure of ZR/BCAR4 cells to lapatinib resulted in modestly increased ERBB2 protein levels. Addition of 4-hydroxytamoxifen to ZR/BCAR4 cells further increased ERBB2 levels, in agreement with earlier observations (Van Agthoven et al, 1994; Bates and Hurst, 1997). In other studies, lapatinib was shown to inhibit phosphorylation of ERBB2 downstream kinases in ERBB2-overexpressing breast cancer (Xia et al, 2002, 2004; Chu et al, 2005; Spector et al, 2005; Konecny et al, 2006). In our model, activity of ERBB2, ERBB3, and the downstream mediators studied are efficiently inhibited in medium containing oestradiol and a low dose of lapatinib. However, cell proliferation was only partially inhibited, again suggesting an escape route via the ER signalling pathway.

EGFR and ERBB2 overexpression is well documented as being involved in tamoxifen resistance (Riggins et al, 2007; Musgrove and Sutherland, 2009; Van Agthoven et al, 2009). EGFR is not involved in our cell models of endocrine resistance because oestrogen-dependent ZR-75-1 and MCF7 cells are devoid of detectable EGFR expression (Van Agthoven et al, 1992), and ERBB2 is present but not overexpressed nor amplified (Hollestelle et al, 2010). Introduction of BCAR4 activates ERBB2 signalling and induces resistance against antioestrogens (Van Agthoven et al, 2012). This suggests that not only ERBB2 overexpression or amplification is associated with tamoxifen resistance, but that the mere activation of the receptor may also have a role in the process. This is in agreement with earlier findings that other models of endocrine resistance, LTED and LTAM cells, showed increased activation of ERBB2 and downstream signalling (Leary et al, 2010). In addition, it has been hypothesised that moderate, as well as low ERBB2 levels, may generate a strong mitogenic signal when the receptor is activated by dimerisation with EGFR or ERBB3 (Frogne et al, 2009).

In ZR/BCAR4 cells cultured with 4-hydroxytamoxifen, the $\mathrm{IC}_{50}$ for lapatinib was approximately $1 \mu \mathrm{m}$. This concentration is achieved in the plasma of patients treated with the recommended daily dose of $1.5 \mathrm{mg}$ (Burris et al, 2005), emphasising the potential feasibility of lapatinib as treatment for antioestrogen resistant breast cancer due to BCAR4 expression. Activated ERBB2 has been found in ER-positive tumours classified as negative for ERBB2 expression according to the standard criteria (Frogne et al, 2009). Moreover, emerging evidence shows that some tumours scoring negative for ERBB2 expression benefit from trastuzumab therapy (Paik et al, 2008; Esteva et al, 2010). At present, treatment with ERBB2-targeted therapies is restricted to patients with breast cancers overexpressing ERBB2. Until now, there are no biomarkers to select patients with ER-positive/ERBB2-negative tumours, which are dependent on ERBB2 signalling (Mayer and Arteaga, 2010), and may benefit from ERBB2-targeted therapies. Although it has been shown that the combination of lapatinib and an aromatase inhibitor is not beneficial for ERBB2-negative, endocrine sensitive or endocrine naive metastatic breast cancer patients (Johnston et al, 2009), this remains to be established for BCAR4-positive tumours.

We have shown that co-expression of $B C A R 4$ and low level of $E R B B 2$ occurs frequently, and that these patients have less benefit from tamoxifen treatment. Although our observations do not prove that the ERBB2 signalling pathway is activated in these tumours, our experimental data suggest that this group might benefit from the combination of lapatinib and antioestrogens. The focus of our future studies will be to determine the phosphorylation status of ERBB2 and downstream mediators on micro tissue 
arrays of a large cohort of breast cancers with known follow-up. Patients with primary tumours with high levels of $E R B B 2$ had the shortest PFS, irrespective of their BCAR4 levels. On the basis of our results it will now be highly relevant to establish whether these $B C A R 4$-positive/ERBB2-low cancers have indeed an activated ERBB2 signalling pathway.

\section{ACKNOWLEDGEMENTS}

We are thankful to GlaxoSmithKline for providing Lapatinib, to Walter Loos for performing the $\mathrm{IC}_{50}$ calculations, Johan Boender for performing the siRNA transfections and Leendert Looijenga for critical reading of the manuscript. This study was funded by Erasmus MC MRACE grant 2007.

\section{Conflict of interest}

JDW: ownership interest and paid consultant for Theranostics Health, LLC. EFP: ownership interest, member of advisory board and unpaid consultant for Theranostics Health, LLC. The remaining authors declare no conflict of interest.

Supplementary Information accompanies the paper on British Journal of Cancer website (http://www.nature.com/bjc)

\section{REFERENCES}

Barone I, Brusco L, Fuqua SA (2010) Estrogen receptor mutations and changes in downstream gene expression and signaling. Clin Cancer Res 16(10): 2702-2708

Bates NP, Hurst HC (1997) An intron 1 enhancer element mediates oestrogen-induced suppression of ERBB2 expression. Oncogene 15(4): 473-481

Brinkman A, Van der Flier S, Kok EM, Dorssers LCJ (2000) BCAR1, a human homologue of the adapter protein p130Cas and ntiestrogen resistance in breast cancer cells. J Natl Cancer Inst 92: 112-120

Burris 3rd HA, Hurwitz HI, Dees EC, Dowlati A, Blackwell KL, O’Neil B, Marcom PK, Ellis MJ, Overmoyer B, Jones SF, Harris JL, Smith DA, Koch KM, Stead A, Mangum S, Spector NL (2005) Phase I safety, pharmacokinetics, and clinical activity study of lapatinib (GW572016), a reversible dual inhibitor of epidermal growth factor receptor tyrosine kinases, in heavily pretreated patients with metastatic carcinomas. J Clin Oncol 23(23): 5305-5313

Carraway KL, Price-Schiavi SA, Komatsu M, Jepson S, Perez A, Carraway CA (2001) Muc4/sialomucin complex in the mammary gland and breast cancer. J Mammary Gland Biol Neoplasia 6(3): 323-337

Carter AM (2007) Animal models of human placentation - a review. Placenta 28(Suppl A): S41-S47

Chu I, Blackwell K, Chen S, Slingerland J (2005) The dual ErbB1/ErbB2 inhibitor, lapatinib (GW572016), cooperates with tamoxifen to inhibit both cell proliferation- and estrogen-dependent gene expression in antiestrogen-resistant breast cancer. Cancer Res 65(1): 18-25

Creighton CJ, Massarweh S, Huang S, Tsimelzon A, Hilsenbeck SG, Osborne CK, Shou J, Malorni L, Schiff R (2008) Development of resistance to targeted therapies transforms the clinically associated molecular profile subtype of breast tumor xenografts. Cancer Res 68(18): 7493-7501

Davies C, Godwin J, Gray R, Clarke M, Cutter D, Darby S, McGale P, Pan HC, Taylor C, Wang YC, Dowsett M, Ingle J, Peto R (2011) Relevance of breast cancer hormone receptors and other factors to the efficacy of adjuvant tamoxifen: patient-level meta-analysis of randomised trials. Lancet 378(9793): 771-784

Di Segni A, Farin K, Pinkas-Kramarski R (2008) Identification of nucleolin as new ErbB receptors- interacting protein. PLoS One 3(6): e2310

Dorssers LCJ, Veldscholte J (1997) Identification of a novel breast-canceranti-estrogen-resistance (BCAR2) locus by cell-fusion-mediated gene transfer in human breast-cancer cells. Int J Cancer 72: 700-705

Esteva FJ, Yu D, Hung MC, Hortobagyi GN (2010) Molecular predictors of response to trastuzumab and lapatinib in breast cancer. Nat Rev Clin Oncol 7(2): 98-107

Frogne T, Laenkholm AV, Lyng MB, Henriksen KL, Lykkesfeldt AE (2009) Determination of HER2 phosphorylation at tyrosine 1221/1222 improves prediction of poor survival for breast cancer patients with hormone receptor-positive tumors. Breast Cancer Res 11(1): R11

Funes M, Miller JK, Lai C, Carraway 3rd KL, Sweeney C (2006) The mucin Muc4 potentiates neuregulin signaling by increasing the cell-surface populations of ErbB2 and ErbB3. J Biol Chem 281(28): 19310-19319

Godinho M, Meijer D, Setyono-Han B, Dorssers LC, van Agthoven T (2011) Characterization of BCAR4, a novel oncogene causing endocrine resistance in human breast cancer cells. J Cell Physiol 226: 1741-1749

Godinho MFE, Sieuwerts AM, Look MP, Meijer D, Foekens JA, Dorssers LCJ, van Agthoven T (2010) Relevance of BCAR4 in tamoxifen resistance and tumour aggressiveness of human breast cancer. $\mathrm{Br} J$ Cancer 103(8): $1284-1291$

Gutierrez MC, Detre S, Johnston S, Mohsin SK, Shou J, Allred DC, Schiff R, Osborne CK, Dowsett M (2005) Molecular changes in tamoxifen-resistant breast cancer: relationship between estrogen receptor, HER-2, and p38 mitogen-activated protein kinase. J Clin Oncol 23(11): 2469-2476

Hollestelle A, Nagel JH, Smid M, Lam S, Elstrodt F, Wasielewski M, Ng SS, French PJ, Peeters JK, Rozendaal MJ, Riaz M, Koopman DG, Ten Hagen TL, de Leeuw BH, Zwarthoff EC, Teunisse A, van der Spek PJ, Klijn JG Dinjens WN, Ethier SP, Clevers H, Jochemsen AG, den Bakker MA, Foekens JA, Martens JW, Schutte M (2010) Distinct gene mutation profiles among luminal-type and basal-type breast cancer cell lines. Breast Cancer Res Treat 121(1): 53-64

Johnston S, Pippen Jr J, Pivot X, Lichinitser M, Sadeghi S, Dieras V, Gomez HL, Romieu G, Manikhas A, Kennedy MJ, Press MF, Maltzman J, Florance A, O'Rourke L, Oliva C, Stein S, Pegram M (2009) Lapatinib combined with letrozole versus letrozole and placebo as first-line therapy for postmenopausal hormone receptor-positive metastatic breast cancer. J Clin Oncol 27(33): 5538-5546

Konecny GE, Pegram MD, Venkatesan N, Finn R, Yang G, Rahmeh M, Untch M, Rusnak DW, Spehar G, Mullin RJ, Keith BR, Gilmer TM, Berger M, Podratz KC, Slamon DJ (2006) Activity of the dual kinase inhibitor lapatinib (GW572016) against HER-2-overexpressing and trastuzumabtreated breast cancer cells. Cancer Res 66(3): 1630-1639

Leary AF, Drury S, Detre S, Pancholi S, Lykkesfeldt AE, Martin LA, Dowsett M, Johnston SR (2010) Lapatinib restores hormone sensitivity with differential effects on estrogen receptor signaling in cell models of human epidermal growth factor receptor 2-negative breast cancer with acquired endocrine resistance. Clin Cancer Res 16(5): 1486-1497

Lipton A, Leitzel K, Ali SM, Demers L, Harvey HA, Chaudri-Ross HA, Evans D, Lang R, Hackl W, Hamer P, Carney W (2005) Serum HER-2/neu conversion to positive at the time of disease progression in patients with breast carcinoma on hormone therapy. Cancer 104(2): 257-263

Malassine A, Frendo JL, Evain-Brion D (2003) A comparison of placental development and endocrine functions between the human and mouse model. Hum Reprod Update 9(6): 531-539

Massarweh S, Schiff R (2006) Resistance to endocrine therapy in breast cancer: exploiting estrogen receptor/growth factor signaling crosstalk. Endocr Relat Cancer 13(Suppl 1): S15-S24

Massarweh S, Schiff R (2007) Unraveling the mechanisms of endocrine resistance in breast cancer: new therapeutic opportunities. Clin Cancer Res 13(7): 1950-1954

Mayer IA, Arteaga CL (2010) Does lapatinib work against HER2-negative breast cancers? Clin Cancer Res 16(5): 1355-1357

Meijer D, Van Agthoven T, Bosma PT, Nooter K, Dorssers LCJ (2006) Functional screen for genes responsible for tamoxifen resistance in human breast cancer cells. Mol Cancer Res 4: 379-386

Mochizuki S, Okada Y (2007) ADAMs in cancer cell proliferation and progression. Cancer Sci 95(5): 621-628

Munzone E, Curigliano G, Rocca A, Bonizzi G, Renne G, Goldhirsch A, Nole F (2006) Reverting estrogen-receptor-negative phenotype in HER-2overexpressing advanced breast cancer patients exposed to trastuzumab plus chemotherapy. Breast Cancer Res 8(1): R4

Musgrove EA, Sutherland RL (2009) Biological determinants of endocrine resistance in breast cancer. Nat Rev Cancer 9(9): 631-643

Paik S, Kim C, Wolmark N (2008) HER2 status and benefit from adjuvant trastuzumab in breast cancer. N Engl J Med 358(13): 1409-1411

Riggins RB, Schrecengost RS, Guerrero MS, Bouton AH (2007) Pathways to tamoxifen resistance. Cancer Lett 256: 1-24 
Scaltriti M, Verma C, Guzman M, Jimenez J, Parra JL, Pedersen K, Smith DJ, Landolfi S, Ramon Y, Cajal S, Arribas J, Baselga J (2009) Lapatinib, a HER2 tyrosine kinase inhibitor, induces stabilization and accumulation of HER2 and potentiates trastuzumab-dependent cell cytotoxicity. Oncogene 28(6): 803-814

Sieuwerts AM, Meijer-van Gelder ME, Timmermans M, Trapman AM, Garcia RR, Arnold M, Goedheer AJ, Portengen H, Klijn JG, Foekens JA (2005) How ADAM-9 and ADAM-11 differentially from estrogen receptor predict response to tamoxifen treatment in patients with recurrent breast cancer: a retrospective study. Clin Cancer Res 11(20): 7311-7321

Spector NL, Xia W, Burris 3rd H, Hurwitz H, Dees EC, Dowlati A, O'Neil B, Overmoyer B, Marcom PK, Blackwell KL, Smith DA, Koch KM, Stead A, Mangum S, Ellis MJ, Liu L, Man AK, Bremer TM, Harris J, Bacus S (2005) Study of the biologic effects of lapatinib, a reversible inhibitor of ErbB1 and ErbB2 tyrosine kinases, on tumor growth and survival pathways in patients with advanced malignancies. J Clin Oncol 23(11): 2502-2512

Tan M, Yu D (2007) Molecular mechanisms of erbB2-mediated breast cancer chemoresistance. Adv Exp Med Biol 608: 119-129

Van Agthoven T, Godinho MFE, Wulfkuhle JD, Petricoin III EF, Dorssers LCJ (2012) Protein pathway activation mapping reveals molecular networks associated with anti-estrogen resistance in breast cancer cell lines. Int $J$ Cancer (in press)

Van Agthoven T, Sieuwerts AM, Meijer-Van Gelder ME, Look MP, Smid M, Veldscholte J, Sleijfer S, Foekens JA, Dorssers LCJ (2009) Relevance of breast cancer antiestrogen resistance genes in human breast cancer progression and tamoxifen resistance. J Clin Oncol 27: 542-549

Van Agthoven T, Sieuwerts AM, Meijer D, Meijer-van Gelder ME, Van Agthoven TLA, Sarwari R, Sleijfer S, Foekens JA, Dorssers LCJ (2010)
Selective recruitment of breast cancer anti-estrogen resistance genes and relevance for breast cancer progression and tamoxifen therapy response. Endocr Relat Cancer 17: 215-230

Van Agthoven T, Van Agthoven TLA, Dekker A, Foekens JA, Dorssers LCJ (1994) Induction of estrogen independence of ZR-75-1 human breast cancer cells by epigenetic alterations. Mol Endocrinol 8: 1474-1483

Van Agthoven T, Van Agthoven TLA, Dekker A, Van der Spek PJ, Vreede L, Dorssers LCJ (1998) Identification of BCAR3 by a random search for genes involved in antiestrogen resistance of human breast cancer cells. EMBO J 17: 2799-2808

Van Agthoven T, Van Agthoven TLA, Portengen H, Foekens JA, Dorssers LCJ (1992) Ectopic expression of epidermal growth factor receptors induces hormone independence in ZR-75-1 human breast cancer cells. Cancer Res 52: 5082-5088

Xia W, Bacus S, Hegde P, Husain I, Strum J, Liu L, Paulazzo G, Lyass L, Trusk P, Hill J, Harris J, Spector NL (2006) A model of acquired autoresistance to a potent ErbB2 tyrosine kinase inhibitor and a therapeutic strategy to prevent its onset in breast cancer. Proc Natl Acad Sci USA 103(20): 7795-7800

Xia W, Liu LH, Ho P, Spector NL (2004) Truncated ErbB2 receptor (p95ErbB2) is regulated by heregulin through heterodimer formation with ErbB3 yet remains sensitive to the dual EGFR/ErbB2 kinase inhibitor GW572016. Oncogene 23(3): 646-653

Xia W, Mullin RJ, Keith BR, Liu LH, Ma H, Rusnak DW, Owens G Alligood KJ, Spector NL (2002) Anti-tumor activity of GW572016: a dual tyrosine kinase inhibitor blocks EGF activation of EGFR/erbB2 and downstream Erk1/2 and AKT pathways. Oncogene 21(41): 6255-6263

This work is published under the standard license to publish agreement. After 12 months the work will become freely available and the license terms will switch to a Creative Commons Attribution-NonCommercial-Share Alike 3.0 Unported License. 